\title{
Layer-by-layer films for tunable and rewritable control of contact electrification
}

\section{Citation}

Soh, Siowling, Xin Chen, Sarah J. Vella, Wonjae Choi, Jinlong Gong, and George M. Whitesides. 2013. "Layer-by-Layer Films for Tunable and Rewritable Control of Contact Electrification." Soft Matter 9(43): 10233.

\section{Published Version}

doi:10.1039/c3sm51983j

\section{Permanent link}

http://nrs.harvard.edu/urn-3:HUL.InstRepos:12361264

\section{Terms of Use}

This article was downloaded from Harvard University's DASH repository, and is made available under the terms and conditions applicable to Open Access Policy Articles, as set forth at http:// nrs.harvard.edu/urn-3:HUL.InstRepos:dash.current.terms-of-use\#OAP

\section{Share Your Story}

The Harvard community has made this article openly available.

Please share how this access benefits you. Submit a story.

Accessibility 


\section{Layer-By-Layer Films for Tunable and Rewritable}

\section{Control of Contact Electrification}

Siowling Soh, ${ }^{a}$ Xin Chen, ${ }^{a}$ Sarah J. Vella,,${ }^{a}$ Wonjae Choi, ${ }^{a}$ Jinlong Gong, ${ }^{a}$ and George M. Whitesides*abc

${ }^{a}$ Department of Chemistry and Chemical Biology, Harvard University, 12 Oxford Street, Cambridge, MA 02138, USA

${ }^{b}$ Wyss Institute for Biologically Inspired Engineering, Harvard University, 60 Oxford Street, Cambridge, MA 02138, USA

${ }^{c}$ Kavli Institute for Bionano Science and Technology, School of Engineering and Applied Sciences, Harvard University, 29 Oxford Street, Cambridge, MA 02138, USA

* Corresponding author: gwhitesides@gmwgroup.harvard.edu 


\section{Table of Content Entry}

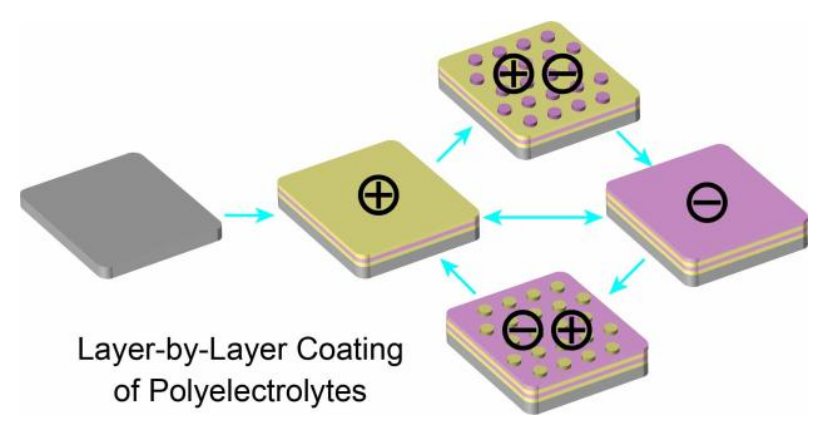

Through depositing or patterning layer-by-layer (LBL) coatings of polyelectrolytes, a surface can be designed that either induces or prevents electrostatic charging in a tunable and rewritable manner during contact electrification. 


\section{Abstract}

Charges generated by contact of solid surfaces (contact electrification) can be hazardous or useful depending on the circumstance. This paper describes a process to design a solid surface rationally to either induce or prevent charging during contact electrification; this process coats the surface with polyelectrolytes. It is observed experimentally that a surface coated with a layer of a polymer having multiple, covalently attached positive charges (a "polycation") develops a positive charge after contacting another surface; a surface coated with a layer of polymer having negative charges (a "polyanion") develops a negative charge. By coating the surface using layerby-layer (LBL) deposition, the tendency of the surface to charge either positively or negatively can be switched: adding a layer of polyelectrolyte with charge opposite to the charge on the surface switches the polarity of the surface. Through microcontact printing $(\mu \mathrm{CP})$, the surface can be stamped to create a mosaic pattern of polycation and polyanion — and importantly, the fraction of the surface area covered with polycation and polyanion can be tuned by using stamps of different patterns. Using poly(diallyldimethylammonium chloride) (PDDA) as the polycation and poly(sodium 4-styrenesulfonate) (PSS) as the polyanion, it is found that for a surface with $>75 \%$ PSS, the surface charges negatively; with $<75 \%$ PSS, the surface charges positively. At $\sim 75 \%$ PSS, the surface becomes non-charging. The patterns on the surface can, subsequently, be erased through coating the surface with a uniform layer of polyelectrolyte. After erasing, the surface is rewritable by depositing or patterning the surface with a desired polyelectrolyte. 
"Contact electrification" is the name given to the set of processes that results in charge separation (that is, generates static electricity) when two solid surfaces are brought into contact and then separated. Processes involving electrostatic charge localized on solid or liquid surfaces are important in many applications; these include electrophotography, ${ }^{1}$ electrostatic precipitation, ${ }^{2}$ powder coating, ${ }^{3}$ and high-voltage, low-current generation (e.g., as in a Van de Graaff generator). The accumulation of electrostatic charge can, however, result in electrical discharges that can cause damage to electronics, explosion of flammable gases and dusts (e.g., during fueling of vehicles or pumping organic liquids; in grain elevators), lightning, and annoyances associated with everyday activities (e.g., sparks while walking on a rug, or charging of clothes while tumbling during drying). ${ }^{4}$ To reduce the electrical potential accumulated by contact electrification, one can either increase (bulk and/or surface) conductivity so that accumulated charge dissipates, or neutralize the charged surfaces with an external source of ions (e.g., with a Zerostat gun). These strategies, however, rely on eliminating the net charge generated after contact electrification, instead of preventing the generation of a net charge on contact.

We demonstrated that it is possible to design surfaces rationally with a desired tendency to charge (or not charge) on contact with other surfaces, by patterning substrates with selfassembled monolayers (SAMs) of molecules containing ionic functional groups. ${ }^{5}$ In this communication, we report the proof of concept of a related but different version of this concept. Using layer-by-layer (LBL) coatings of polyelectrolytes, we can either induce or prevent charging of surfaces in a tunable and rewritable manner.

Although the mechanisms governing contact electrification are still incompletely understood, ${ }^{4,6-8}$ it is probable that charge separation on contact of insulating with insulating materials, and of insulating with metallic materials, involves predominantly the movement of 
ions rather than electrons (although electrons are certainly involved in some spontaneous discharge events). ${ }^{6,9}$ In particular, for two solid surfaces covered with covalently bound, fixed, ionic functional groups and mobile counterions, it is clear that charge separation results from the transfer of mobile counterions between the surfaces. ${ }^{10-12}$ For example, polystyrene surfaces that are chemically modified with sulfonic (or quaternary amine) groups charge negatively (or positively) when brought into contact with polyethylene - an observation that is in accordance to the ion-transfer mechanism. In a similar demonstration, polymers that contain bonded cations acquire a positive charge, whereas polymers that contain bonded anions acquire a negative charge, during contact electrification. ${ }^{13}$

To test our understanding of the ion-transfer mechanism, we fabricated patterned SAMs of charged silanes (in a previous work ${ }^{5}$ ), to control charging of glass surfaces. Specifically, we made a surface that consisted of negatively charging, plasma-oxidized glass, with a fraction of the surface patterned with positively charged silanes. When the fraction of the patterned surface was $>50 \%$, the surface charged positively; when the fraction was $<50 \%$, the surface charged negatively. Importantly, when the fraction was 50\%, the surface was non-charging. More recently, Thomas and coworkers have demonstrated that UV radiation can be used to switch the charging of solid surfaces between two modes (charging either positively or negatively), after functionalizing the surface with a photo-sensitive molecule (a spiropyran-based photochromic polymer). ${ }^{14}$

This paper demonstrates the control of contact electrification in dielectric materials using thin films of polyelectrolytes prepared by layer-by-layer (LBL) deposition. LBL assembly is a bottom-up fabrication technique that forms integrated thin films comprising alternating layers of materials carrying complementary charges or functional groups. ${ }^{15-17}$ The most common examples of LBL assembly use films of polyelectrolytes. Adsorption of a polyelectrolyte layer 
onto the surface of a substrate, where the polyelectrolyte carries a fixed charge opposite to that of the substrate, generates the first layer of an LBL film. In practice, plasma treatment of many substrates (including most metals, oxides, and polymers) generates negatively charged surfaces; therefore, the first layer in a LBL system is often a polyelectrolyte that carries a fixed positive charge. Adsorption of a second polyelectrolyte carrying charge that is opposite to the charge of the first layer of polyelectrolyte (but same polarity as the substrate) forms the second layer. ${ }^{15}$ Each additional layer flips the polarity of the charge on the surface; repeating these steps multiple times creates a multilayered LBL film.

LBL is a versatile technique: it is possible to deposit many different types of polymers on many different substrates; these include most organic polymers, inorganic materials (e.g., glass/silicon), and metals/metal oxides. ${ }^{18}$ Various types of functional materials, ranging from small molecules (e.g., ions, organic dyes), to macromolecules (e.g., polymers, proteins, DNAs), to molecular assembles (e.g., nanodots, nanotubes), and complex biological entities (e.g., virus), have also been successfully integrated into LBL films. ${ }^{15}$ It is also possible to selectively deposit polyelectrolytes on specific regions of a surface through micro-contact printing $(\mu \mathrm{CP}){ }^{19}$ this technique may be useful for commercially relevant functionalities (e.g., for the microfabrication of flexible electronics or complex biosurfaces). ${ }^{20}$

By depositing an LBL film onto a dielectric material, we are able to control both the polarity and magnitude of the charge developed when the surface is brought into contact with another surface. The charge developed on such a surface can be tuned to be highly positive by depositing a polycation as the outermost layer, or highly negative by depositing a polyanion as the outermost layer. By using micro-contact printing $(\mu \mathrm{CP})$, we generated an outermost layer comprising different area fractions of polycation and polyanion in a mosaic pattern. Importantly, the average charge developed on this surface can be tuned to any value between the two 
extremes attainable by either a uniform layer of polycation or polyanion as the outermost layer. This method can also produce a virtually non-charging surface.

The LBL surface is reversibly switchable. When a substrate with a uniform LBL coating is covered with a polyelectrolyte solution of the opposite polarity, the substrate switches its polarity. The LBL surface is also erasable and rewritable. When a substrate patterned with both types of polyelectrolytes is covered with a solution containing one of the polyelectrolytes, the substrate generates a uniform outermost layer terminated with the polyelectrolyte in the solution — the pattern on the surface is thus erasable. After erasing the pattern, it is then possible to coat the substrate with a desired layer of polyelectrolyte or a pattern of both types of polyelectrolytes — the surface is rewritable.

This technique for LBL-based coating on solid surfaces offers six advantages for controlling contact electrification of the surface: i) it is simple; ii) the process used to coat the LBL films can be automated, and can be completed relatively quickly ( $\sim$ min per layer in some procedures $){ }^{21}$ iii) the charge generated on the surface is erasable through coating the surface uniformly with a polyelectrolyte (using LBL deposition) of the desired charge; iv) the charge is tunable, or can be patterned, by printing different regions of the surface with polycations and polyanions using $\mu \mathrm{CP}$ or other techniques; v) the technique is applicable to a variety of polar surfaces; vi) large-area LBL coating can be accomplished economically using spray-coating technology, ${ }^{22}$ and printed (we presume) by reel-to-reel techniques.

The polycation used in our experiments was poly(diallyldimethylammonium chloride) (PDDA, M.W. 400,000 500,000) and the polyanion used was poly(sodium 4-styrenesulfonate) (PSS, M.W. 70,000). We chose these two polyelectrolytes because both have high charge densities and are so-called "strong" polyelectrolytes. LBL films formed by a pair of strong polyelectrolytes are 
typically very stable, uniform, and are made of very thin ( a few nanometers) layers. ${ }^{23} \mathrm{We}$ followed procedures largely developed and described by Hammond and coworkers to prepare LBL-coated surfaces (Fig. 1). ${ }^{24}$ Briefly, we first cleaned a polystyrene substrate (VWR Petri dish) with de-ionized water, and exposed the substrate to an air plasma to introduce negatively charged groups (probably due to carboxylic groups). ${ }^{20,25}$ When this surface was covered with a PDDA solution in water ( $2 \mathrm{mM}$, based on repeat units) for 30 minutes, the positively charged PDDA polymer chains adsorbed spontaneously onto the surface. ${ }^{20}$ Covering the substrate with a PSS ( $2 \mathrm{mM}$, based on repeat units) solution allowed the negatively charged PSS polymer to adsorb onto the surface. The process could be repeated to form layer-by-layer films on the substrate by covering the substrate alternatively with PDDA and PSS solutions. Finally, we rinsed the substrate with $2 \mathrm{mM} \mathrm{NaCl}$ solution $(50 \mathrm{~mL})$ three times, dried it with a stream of nitrogen, and stored it in a vacuum oven $\left(1\right.$ torr, $\left.55^{\circ} \mathrm{C}\right)$ overnight before measuring the charge of the surface

We used the rolling sphere tool (RST), a system first developed by Grzybowski et al., to measure the kinetics of contact charging. ${ }^{26-28}$ Briefly, in this type of measurement, a rotating bar magnet caused a ferromagnetic steel sphere $(d=3.2 \mathrm{~mm})$ to roll along a circular path (periodicity $=0.5 \mathrm{~s}$ ) on an insulating substrate (Fig. 2a). We cleaned the spheres extensively by agitating them in acetone, water (Millipore), and ethanol as reported in our earlier publication, ${ }^{29}$ and exposed them to an air plasma for 30 seconds before the experiments. An aluminum electrode (width $=1 \mathrm{~cm}$ ), located directly beneath the substrate and connected to an electrometer, measured the charge on the portion of the substrate near the electrode, $Q_{\text {sne }}$. For every revolution that the sphere passed over the electrode, the electrometer also recorded the net charge of the sphere and the substrate near the electrode, $Q_{\mathrm{sp}}+Q_{\text {sne. }}$ This setup provided a real-time, noninvasive way to monitor the kinetics of contact charging of the sphere and the substrate. We 


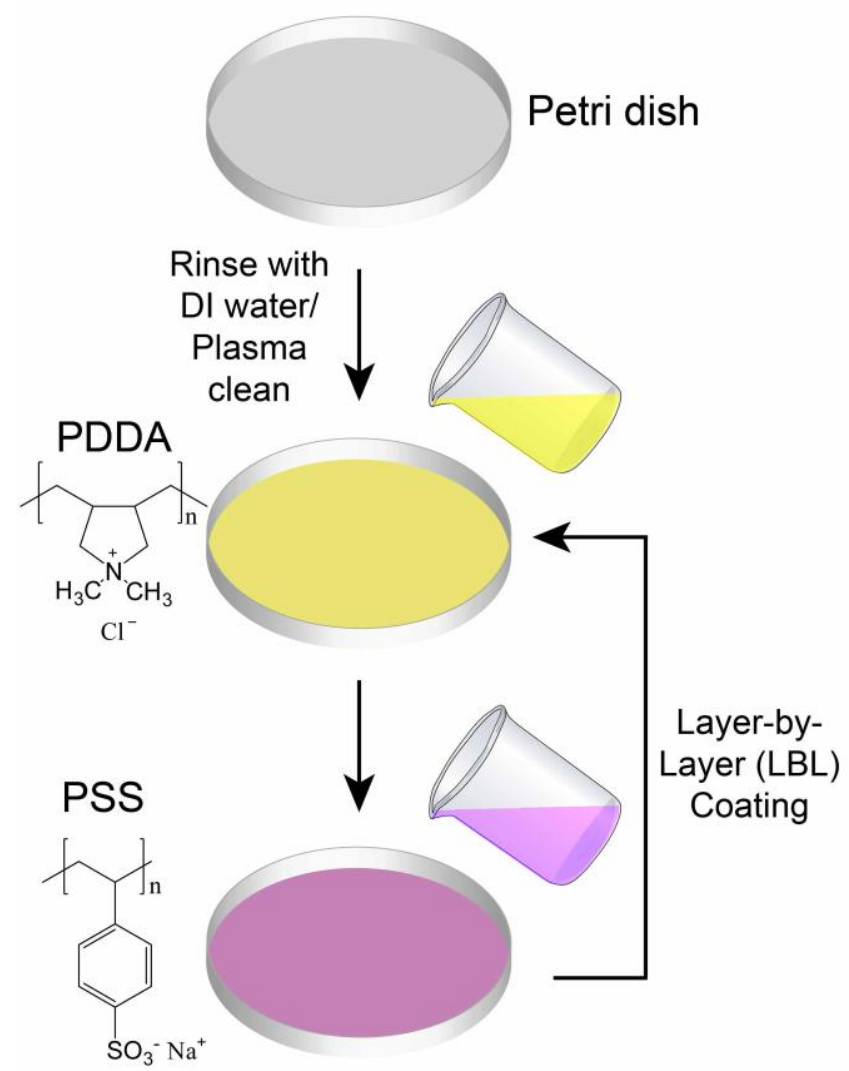

Fig. 1 Illustration of coating polyelectrolytes layer-by-layer (LBL) on the surface of a Petri dish. The Petri dish is first rinsed with de-ionized water and plasma cleaned before coating with a layer of polycation (e.g., PDDA) and a layer of polyanion (e.g., PSS). Thicker LBL films, or films with multiple (e.g., more than two) types of polymer, can be made by repeatedly coating the surface with layers of polycations and polyanions. 
a)
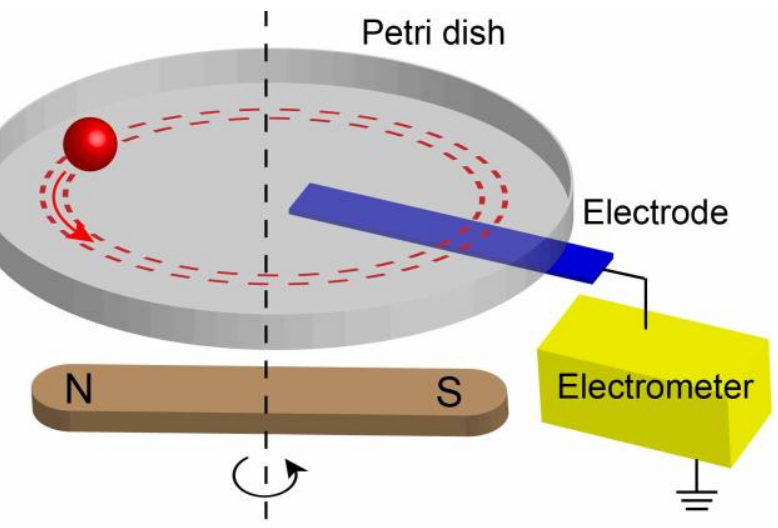

b)

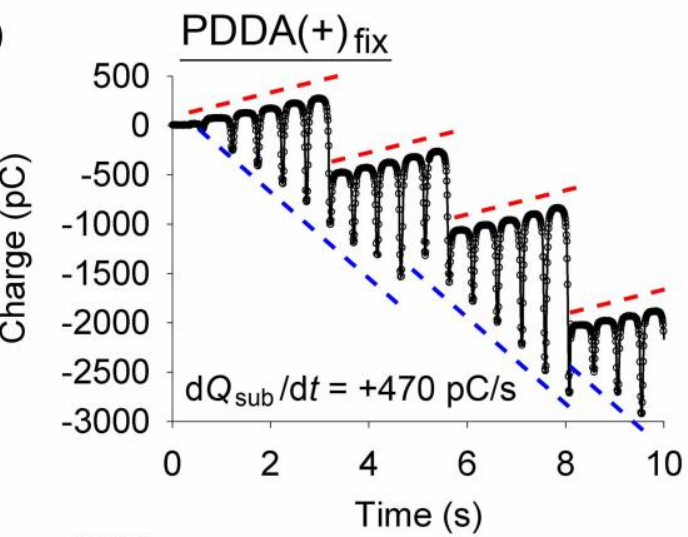

c) 2000

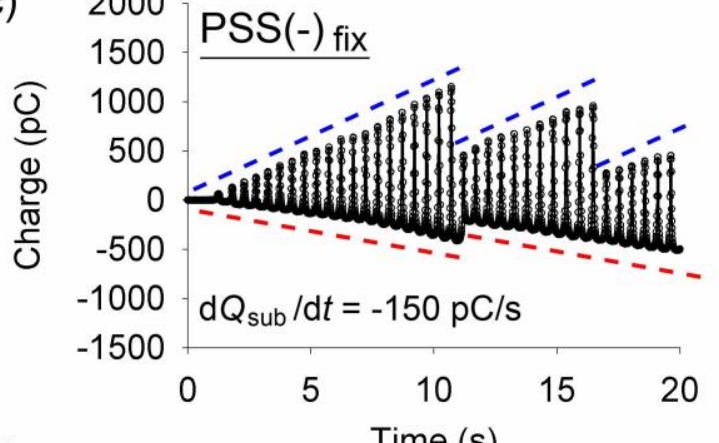

d)

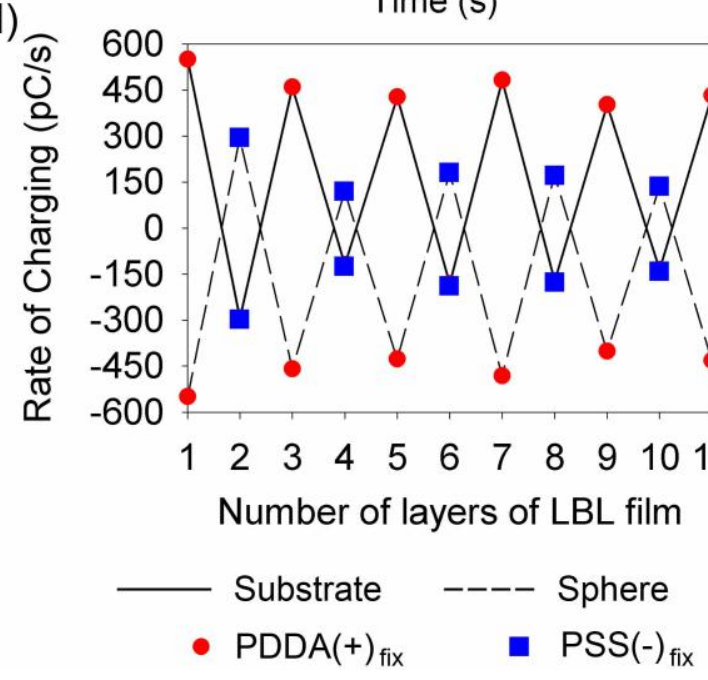


Fig. 2 Contact electrification between a stainless steel sphere and a substrate covered with uniform LBL coatings of polyelectrolytes. (a) Illustration of the Rolling Sphere Tool (RST) used to monitor the contact-charging process between the sphere and the substrate. (b) An LBL film terminated with a uniform layer of PDDA charged positively (and the sphere charged negatively). (c) An LBL film terminated with a uniform layer of PSS charged negatively (and the sphere charged positively). The net rate of charging of the sphere can be determined by the difference between the slopes of the two enveloping lines (red and blue dash lines). According to our definition, the rate of charging of the substrate, $\mathrm{d} Q_{\text {sub }} / \mathrm{d} t$, is positive if the red line is above the blue line, and negative if the red line is below the blue line. The plot in (c) has a longer time axis than (b) since the rate of charging is slower in (c) than in (b). (d) The magnitude and polarity of the rate of charging changed back and forth with every additional layer deposited on the LBL film, but remained largely unchanged for the surfaces terminated with the same electrolyte (i.e., every other layer). 
performed all the RST experiments in a hermetic chamber, where the temperature inside the chamber was controlled at $22-23{ }^{\circ} \mathrm{C}$, and the relative humidity was within the range of $10-20 \%$. Charging curves such as those plotted in Fig. $2 \mathrm{~b}$, can be characterized largely by two straight lines (we have described the analysis of these lines in detail previously ${ }^{28,29}$ ). The red dashed line describes the charge developed on the portion of the substrate near the electrode, $Q_{\text {sne, }}$ and the blue dashed line describes the net charge of (the same portion of) the substrate and the sphere, $Q_{\text {sne }}+Q_{\text {sp. }}{ }^{28,29}$ The difference between the two lines at each point in time gives the charge developed on the sphere, $Q_{\mathrm{sp}}$. The fact that these two lines are straight — and as a result, the difference between them is a straight line — indicates that the rate of charge separation is linear for the system presented in this work. The difference in the slopes of the two lines represents the rate of charging of the sphere, $\mathrm{d}\left(Q_{\mathrm{sp}}\right) / \mathrm{d} t$. As the sphere revolves around the substrate, an annular portion of the substrate is in contact with the rolling sphere (red dashed lines in Fig. 2a). According to the conservation of charge during contact electrification, the rate of charging of this annular portion of the substrate in contact with the rolling sphere $\mathrm{d}\left(Q_{\text {sub }}\right) / \mathrm{d} t$, is equal in magnitude, but opposite in polarity, to the rate of charging of the sphere, $\mathrm{d}\left(Q_{\mathrm{sub}}\right) / \mathrm{d} t=-\mathrm{d}\left(Q_{\mathrm{sp}}\right) / \mathrm{d} t$. In this study (and in one of our previous studies ${ }^{5}$ ), we report the rate of charging of the substrate, $\mathrm{d}\left(Q_{\text {sub }}\right) / \mathrm{d} t$, to indicate the rate at which the substrate charges with the rolling sphere. We choose to report $\mathrm{d}\left(Q_{\text {sub }}\right) / \mathrm{d} t$ instead of the maximum amount of charge developed when the substrate charges with the rolling sphere because it is difficult to measure the maximum storable charge on the substrate reproducibly. The maximum amount of charge storable on a material is influenced by discharge, and discharge is — to some extent — idiosyncratic. ${ }^{30}$ When the measured charge on the rolling sphere reached $\sim 900 \mathrm{pC}$, discontinuities occurred in the charging curves (such as those in Fig. 2b-c). We attribute these features to spontaneous electrical discharges through ambient air. ${ }^{29,30}$ According to Equation 1, where $E$ is the electric field, $\varepsilon_{0}$ is the permittivity, and 
$r$ is the radius of the rolling sphere, the electric field generated by $900 \mathrm{pC}$ on the surface of a sphere with a diameter of $3.2 \mathrm{~mm}$ would exceed the dielectric breakdown limit of air $(\sim 3$ $\mathrm{MV} / \mathrm{m})^{30}$

$$
E=\frac{Q}{4 \pi \varepsilon_{0} r^{2}}
$$

Fig. $2 b$ and $2 c$ plot the representative charging curves. The rates of charging are +470 $\mathrm{pC} / \mathrm{sec}$ for the PDDA $(+)_{\mathrm{fix}}$ terminated and $-150 \mathrm{pC} / \mathrm{sec}$ for the PSS $(-)_{\text {fix }}$ terminated films. We assume that the polyelectrolyte backbones on these LBL films are anchored on the surfaces during contact electrification, but the small counterions are mobile and can transfer from one surface to another. In our experiments, $\mathrm{Cl}^{-}$ions are presumably the major mobile counterions on PDDAterminated films and $\mathrm{Na}^{+}$ions are the major mobile counterions on PSS-terminated films. We designed our system on the hypothesis that the dominant charge carriers in our experiments are the mobile counterions present only on the outermost layer. Experimentally, the charge developed on the surface during contact electrification has the same polarity as that of the polyelectrolyte on the outermost layer; this observation is consistent with the ion-transfer mechanism for contact electrification. ${ }^{6,10-12}$ The absolute magnitude of the rate of charging is different for PDDA- and PSS-terminated films; this difference may be due to a faster rate of transfer of $\mathrm{Cl}^{-}$ions than $\mathrm{Na}^{+}$ ions from the substrate to the rolling sphere. We cannot, however, rule out contributions from other ions (e.g., hypothetically hydroxide or hydronium). ${ }^{6}$

The plot in Fig. $2 \mathrm{~d}$ shows the changes in the rate of charging of the substrate and the sphere with each additional layer of the polyelectrolytes coated on the substrate - importantly, the substrate and the sphere continue to switch their polarity for multiple (eleven) layers of LBL coatings. Not surprisingly, the rate of charging of the LBL films is similar for films terminated with the same type of polyelectrolyte (i.e., $~ 450 \mathrm{pC} / \mathrm{s}$ for PDDA-terminated and $\sim 150 \mathrm{pC} / \mathrm{s}$ for 
PSS-terminated films) at every other layer since the properties (e.g., electrostatic) of these strong polyelectrolyte LBL films are not expected to change from layers to layers. ${ }^{15}$

We used micro-contact printing $(\mu \mathrm{CP})$ to produce LBL films with a mosaic of PDDA and PSS regions as the outermost layer (Fig. 3). The method used for $\mu \mathrm{CP}$, and the experimental conditions used to pattern LBL films, were similar to those used by Hammond and coworkers. ${ }^{19}$ First, we fabricated three PDMS stamps with different areas of contact (i.e., $25 \%, 50 \%$ or $75 \%$ of the entire surface of the substrate) between the stamp and the substrate (Fig. S1 in the supporting material shows the patterns of these stamps). The features on the stamps were small $(\sim 500-700$ $\mu \mathrm{m}$ for all three stamps) compared to the size of the substrate $(10 \mathrm{~cm}$ in diameter $)$. Air-plasma treatment (30 seconds) rendered the surfaces of the stamp hydrophilic. Hydrophilic surfaces were necessary to stamp aqueous solutions of polyelectrolytes onto the substrate. We prepared LBL films on the substrate using the method described earlier. As an example (see Fig. 3), a stamp containing a solution of PSS ( $5 \mathrm{mM}$, based on repeat unit) transferred the PSS polymer chains onto the substrate when the stamp was gently pressed against a PDDA-terminated LBL film. We left the stamp in contact with the substrate for 30 minutes. ${ }^{31}$ During the $\mu \mathrm{CP}$ process, the regions on the substrate in contact with the stamp were coated with an additional layer of the polyelectrolyte on the stamp (e.g., PSS for the case illustrated in Fig. 3), while the regions not in contact with the stamp remained unchanged (remained as PDDA). ${ }^{19}$ This process created a mosaic of PDDA- and PSS-terminated regions on the surface of the substrates. After removing the stamp, we rinsed and dried the substrate using the same methods we used to prepare uniform films.

Fig. 4 plots representative charging curves of these patterned films, and reports their rates of charging, $\mathrm{d}\left(Q_{\mathrm{sub}}\right) / \mathrm{d} t$. As expected, the area fraction of PDDA- and PSS-terminated regions on the surface of the substrate determines the overall rate of charging. For example, the rate of 


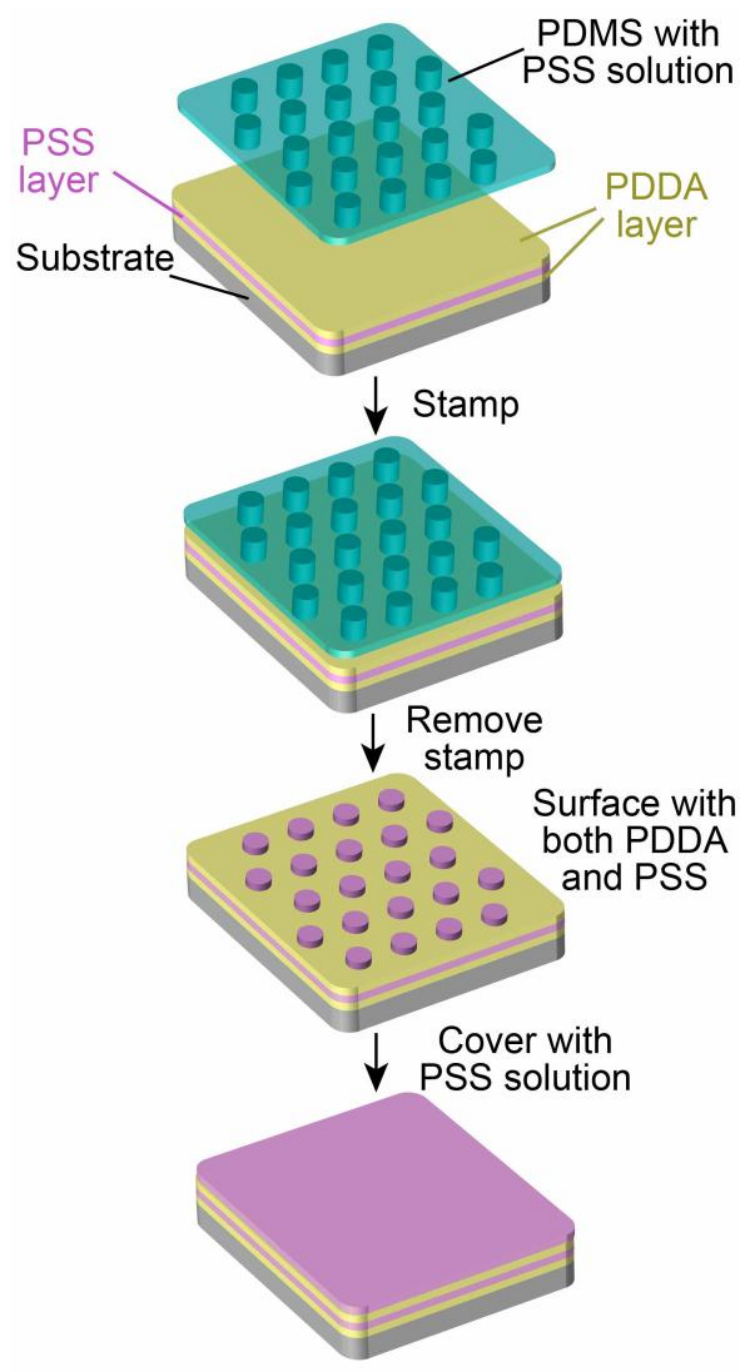

Fig. 3 Schematic representation of micro-contact printing $(\mu \mathrm{CP})$, followed by coating the surface with a uniform layer of polyelectrolyte. The yellow and pink layers represent PDDA and PSS respectively. In this illustration, the LBL film consists of a uniform layer of PDDA as its outmost layer before stamping. After stamping with PDMS (patterned with circular posts) filled with PSS solution, the outermost surface becomes a film terminated with a mosaic of PDDA and PSS; the rate of charging of this mosaic surface is the weighted average between the two extremes, where the surface contains either a uniform layer of PDDA or PSS. Covering the entire surface of the substrate with PSS solution erases the pattern on the outermost layer, and results in a uniform PSS-terminated film. 

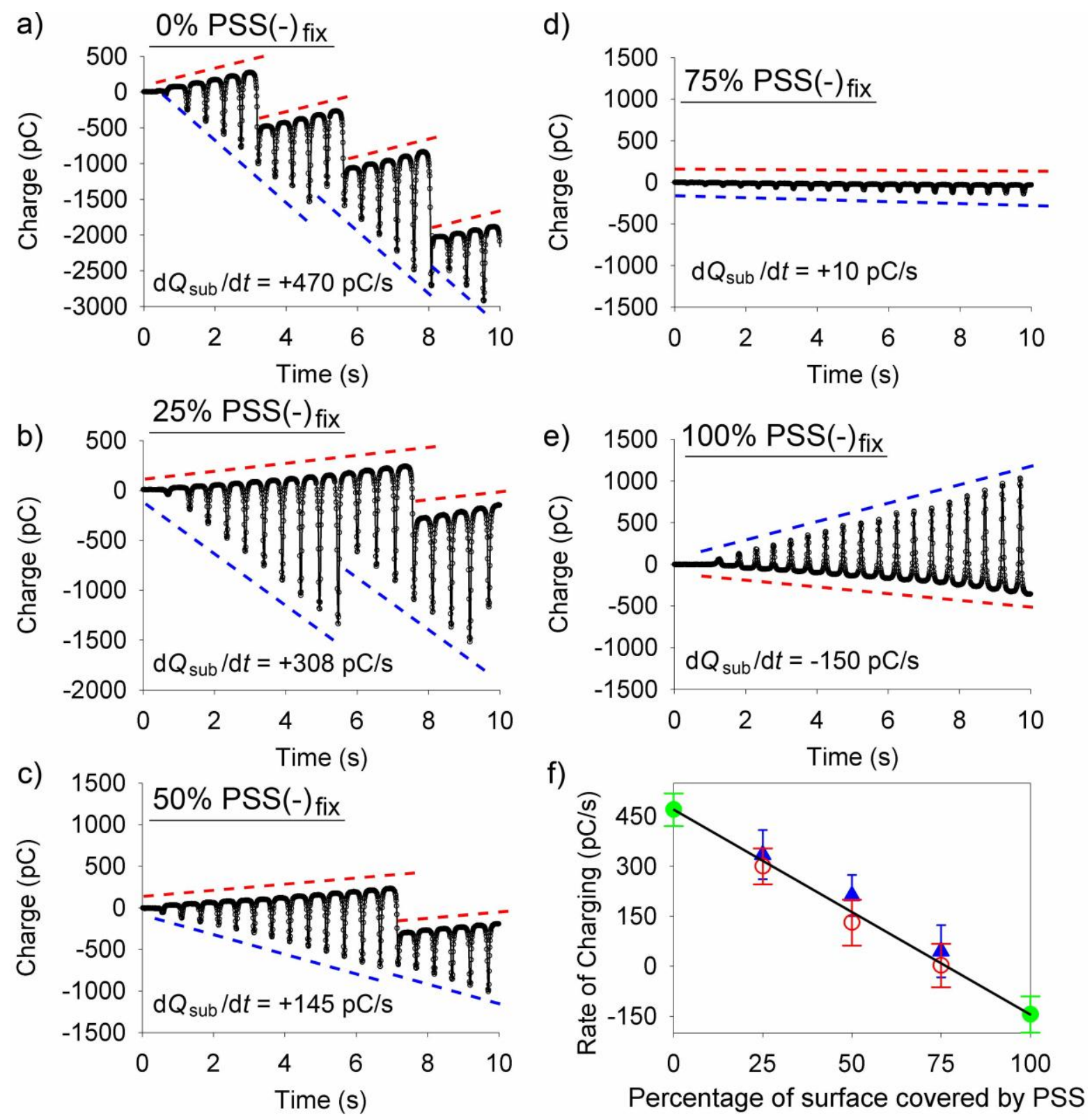
Fig. 4 Charges and rates of charging of LBL films with surfaces patterned with different area fractions of PSS. (a-e) Real-time measurements of charge for LBL films with PSS covering 0\%, $25 \%, 50 \%, 75 \%$, and $100 \%$ of the entire surface area of the substrate. At $75 \%$ coverage of PSS, the surface charges slowly $(+10 \mathrm{pC} / \mathrm{s})$, and is effectively a non-charging surface. (f) The rate of charging of the substrate depends linearly on the fraction of the surface covered by PSS and PDDA. Open circles (red) represent rates of charging after stamping PSS on PDDA-terminated films; the solid triangles (blue) represent rate of charging of the substrate after stamping PDDA on PSS-terminated films. These two sets of data are indistinguishable within experimental uncertainty ( $n=4$ for all experiments; the error bars are approximations of the standard deviation). 
charging of a 50-50 mosaic layer (Fig. 4c) is approximately the average value of the two limiting cases shown in Fig. 2b and 2c-a uniform PDDA-terminated and a uniform PSS-terminated layer respectively. By tuning the area fraction of the regions covered by the two polyelectrolytes, the overall rate of charging can be tuned to any value between the two limiting cases (i.e., any value between $+470 \mathrm{pC} / \mathrm{s}$ and $-150 \mathrm{pC} / \mathrm{s}$ in our system). A special case of particular importance is a non-charging surface. In our system, a surface coverage of 25\% PDDA and 75\% PSS produces an approximately non-charging surface. As shown in Fig. 4d, the rate of charging of such a surface is $10 \mathrm{pC} / \mathrm{s}$, which is more than one order of magnitude smaller than LBL films coated uniformly with either type of polyelectrolyte $(+470 \mathrm{pC} / \mathrm{s}$ for PDDA-terminated and -150 $\mathrm{pC} / \mathrm{s}$ for PSS-terminated films). In other words, the LBL method of coating a surface provides a general strategy to create a virtually non-charging surface. The pattern formed by both types of polyelectrolytes on the surface is erasable. For example, the final step in Fig. 3 illustrates the case in which covering a patterned surface with PSS solution generates a uniform LBL film, with PSS as the outermost layer. LBL deposition is a self-limiting process; electrostatic repulsion prevents PSS from depositing on regions already covered by PSS, and fresh PSS deposits only on regions with exposed PDDA (i.e., regions not previously stamped with PSS). After generating a surface with a uniform coat of polyelectrolyte, the rate of charging returned to the value expected for a uniform PSS-terminated film.

Fig. 4f shows a linear dependence between the rate of charging of the substrate, $\mathrm{d}\left(Q_{\text {sub }}\right) / \mathrm{d} t$, and the fraction of the surface covered by PSS. Open circles (red) represent the rate of charging of substrates generated by stamping PSS on PDDA-terminated films. The opposite type of procedure can also be followed: the solid triangles (blue) represent the rate of charging of the substrates generated by stamping PDDA on uniform PSS-terminated films. These two sets of data are indistinguishable within experimental uncertainty, and thus confirm that the overall rate 
of charging of the surface is determined only by the area fraction of the PSS- and PDDAterminated areas.

We show that the outermost layer of a LBL film controls contact electrification and the transfer of charge between the surface of the film and a rolling steel sphere. These results support the arguments for ion transfer as the dominant mechanism of contact electrification in systems comprising polyelectrolytes; ${ }^{6}$ our observations can be rationalized by assuming that the small, mobile counterions (either $\mathrm{Cl}^{-}$or $\mathrm{Na}^{+}$ions in our system) are the main charge carriers.

The capabilities of a LBL-coated surface are: (1) the polarity and the charge on the surface can be reversibly switched by depositing additional layers of polyelectrolytes. (2) The surface can be tuned to charge either positively or negatively (or not to charge) by patterning the surface into regions exposing both types of polyelectrolytes. (3) The pattern on a patterned surface can be erased when it is covered with a solution containing one of the polyelectrolytes. (4) The surface — with patterns erased — can be rewritten with a new pattern when it is coated and/or patterned again with a new layer of polyelectrolyte.

In short, coating and patterning LBL films on a surface provide a flexible method to create a surface with a desired direction (polarity) and capacity for contact electrification. Plausibly, these LBL-coated surfaces should be valuable as designed materials that resist electrical charging and, consequently, discharging. As a potential example of an application, it may be possible to create a surface (e.g., of a conveyor belt) that would prevent charging of particulate granular solids (e.g., polymeric particles, or pharmaceutical powders) during contact with the belt. According to the triboelectric series (a list of materials organized according to their affinity to charge either positively or negatively ${ }^{32}$ ), Nylon, for example, has a tendency to charge positively against many other solid surfaces. In order for Nylon particles not to charge 
significantly, it is necessary to design a surface with similar affinity to charge positively, and one approach to such a designer surface might be to pattern the surface with different types of polyelectrolytes so that the affinity for charge could be tuned to be similar to Nylon.

It might also be useful to have the flexibility to rewrite and tune the affinity for charge of a surface according to the environment or application. Many external factors affect contact electrification: examples include the humidity of the atmosphere and the temperature, ${ }^{33}$ differences in the pressure applied in contacting the surfaces, and differences in the way the surface are contacted (e.g., by rubbing, or sliding, or ostensibly non-frictional contact). ${ }^{34}$ It might, therefore, be useful to be able to erase, rewrite, and adjust the surface in order to adapt to varying external factors. Combined with the capability to coat large areas with LBL films rapidly using the spraying technique developed recently, ${ }^{22}$ we believe that this method to change the affinity of the surface for charge (either positively or negatively) has the potential to be applicable to largescale processes.

\section{Acknowledgements}

This work was supported by the U.S. Department of Energy, Office of Basic Energy Sciences, Division of Materials Sciences and Engineering under award \# ER45852.

\section{References:}

(1) L. B. Schein, J. Electrost., 1999, 46, 29.

(2) P. A. Lawless and R. F. Altman. Electrostatic Precipitators. In Wiley Encyclopedia of Electrical and Electronics Engineering; Webster, J. G., Ed.; John Wiley \& Sons, Inc, 1999.

(3) J. F. Hughes Electrostatic Powder Coating; Research Studies Press: New York, 1984. 
(4) D. J. Lacks and R. M. Sankaran, J. Phys. D-Appl. Phys., 2011, 44, 453001.

(5) S. W. Thomas, S. J. Vella, M. D. Dickey, G. K. Kaufman and G. M. Whitesides, J. Am. Chem. Soc., 2009, 131, 8746.

(6) L. S. McCarty and G. M. Whitesides, Angew. Chem. Int. Edit., 2008, 47, 2188.

(7) H. T. Baytekin, A. Z. Patashinski, M. Branicki, B. Baytekin, S. Soh and B. A. Grzybowski, Science, 2011, 333, 308.

(8) H. T. Baytekin, B. Baytekin, S. Soh and B. A. Grzybowski, Angew. Chem. Int. Edit., $2011, \mathbf{5 0}, 6766$.

(9) S. Piperno, H. Cohen, T. Bendikov, M. Lahav and I. Lubomirsky, Angew. Chem. Int. Edit., 2011, 50, 5653.

(10) L. S. McCarty, A. Winkleman and G. M. Whitesides, J. Am. Chem. Soc., 2007, 129, 4075.

(11) L. S. McCarty, A. Winkleman and G. M. Whitesides, Angew. Chem. Int. Edit., 2007, 46, 206.

(12) A. F. Diaz and D. Fenzel-Alexander, Langmuir, 1993, 9, 1009.

(13) D. Fenzel-Alexander, P. Brock and A. Diaz, Langmuir, 1994, 10, 3323.

(14) S. Friedle and S. W. Thomas, Angew. Chem. Int. Edit., 2010, 49, 7968.

(15) G. Decher, Science, 1997, 277, 1232.

(16) M. Ferreira, J. H. Cheung and M. F. Rubner, Thin Solid Films, 1994, 244, 806.

(17) Multilayer Thin Films: Sequential Assembly of Nanocomposite Materials; 2nd ed.; G.

Decher and J. B. Schlenoff, Eds.; Wiley-VCH: Weinheim, 2012.

(18) P. T. Hammond, Adv. Mater., 2004, 16, 1271.

(19) P. T. Hammond and G. M. Whitesides, Macromolecules, 1995, 28, 7569.

(20) J. Park and P. T. Hammond, Adv. Mater., 2004, 16, 520. 
(21) K. C. Krogman, N. S. Zacharia, S. Schroeder and P. T. Hammond, Langmuir, 2007, 23, 3137.

(22) K. C. Krogman, J. L. Lowery, N. S. Zacharia, G. C. Rutledge and P. T. Hammond, Nat. Mater., 2009, 8, 512.

(23) G. Decher, J. D. Hong and J. Schmitt, Thin Solid Films, 1992, 210, 831.

(24) K. C. Wood, J. Q. Boedicker, D. M. Lynn and P. T. Hammond, Langmuir, 2005, 21, 1603.

(25) Z. W. Ma, W. He, T. Yong and S. Ramakrishna, Tissue Eng., 2005, 11, 1149.

(26) J. A. Wiles, B. A. Grzybowski, A. Winkleman and G. M. Whitesides, Anal. Chem., 2003, 75, 4859 .

(27) B. A. Grzybowski, M. Fialkowski and J. A. Wiles, J. Phys. Chem. B, 2005, 109, 20511.

(28) S. J. Vella, X. Chen, S. W. Thomas, X. H. Zhao, Z. G. Suo and G. M. Whitesides, J. Phys. Chem. C, 2010, 114, 20885.

(29) S. W. Thomas, S. L. Vella, G. K. Kaufman and G. M. Whitesides, Angew. Chem. Int. Edit., 2008, 47, 6654 .

(30) S. Soh, S. W. Kwok, H. Liu and G. M. Whitesides, J. Am. Chem. Soc., 2012, 134, 20151.

(31) We used 30 minutes for stamping the polyelectrolytes since it was the same amount of time used for coating uniform layers of LBL films as described earlier. A previous study reported a much shorter stamping time (30 secs) for patterning LBL films by using a higher concentration of the polyelectrolytes (ref: M. C. Berg, J. Choi, P. T. Hammond and M. F. Rubner, Langmuir, 2003, 19, 2231).

(32) A. F. Diaz and R. M. Felix-Navarro, J. Electrost., 2004, 62, 277.

(33) W. D. Greason, J. Electrost., 2000, 49, 245.

(34) J. Lowell and A. C. Roseinnes, Adv. Phys., 1980, $29,947$. 
Supporting Information for

\section{Layer-By-Layer Films for Tunable and Rewritable}

\section{Control of Contact Electrification}

Siowling Soh, ${ }^{a}$ Xin Chen, ${ }^{a}$ Sarah J. Vella, ${ }^{a}$ Wonjae Choi, ${ }^{a}$ Jinlong Gong, ${ }^{a}$ and George M. Whitesides* $a b c$

${ }^{a}$ Department of Chemistry and Chemical Biology, Harvard University, 12 Oxford Street, Cambridge, MA 02138, USA

${ }^{b}$ Wyss Institute for Biologically Inspired Engineering, Harvard University, 60 Oxford Street, Cambridge, MA 02138, USA

${ }^{c}$ Kavli Institute for Bionano Science and Technology, School of Engineering and Applied Sciences, Harvard University, 29 Oxford Street, Cambridge, MA 02138, USA

* Corresponding author: gwhitesides@gmwgroup.harvard.edu 
Fig. S1. Schematic of the features on the three PDMS stamps used for patterning polyelectrolytes on the substrate. The patterns on the stamps are fabricated according to the percentage of the area of contact between the stamp and the substrate (protruding regions, as indicated in light blue in the scheme) over the entire surface area of the substrate. Panels a, b, and c show patterns capable of stamping $25 \%, 50 \%$, and $75 \%$ of the surface of the substrate. The first two patterns consist of circular posts with different diameters arranged in a hexagonal layout. The last pattern is the reverse of the first pattern.

a)

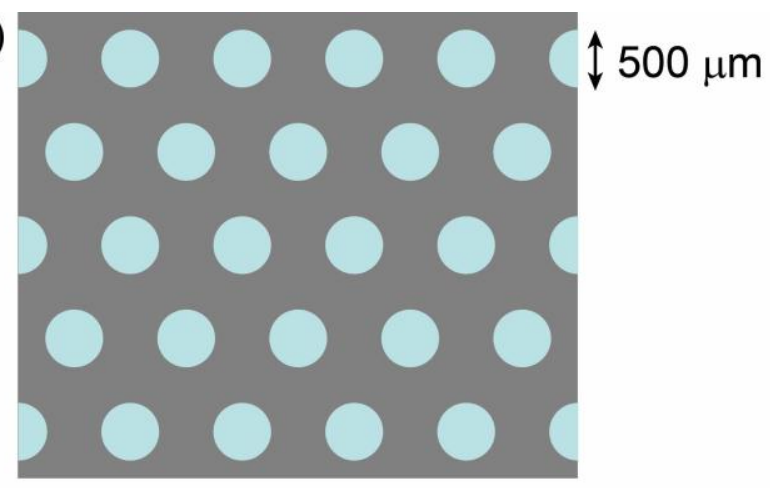

b)

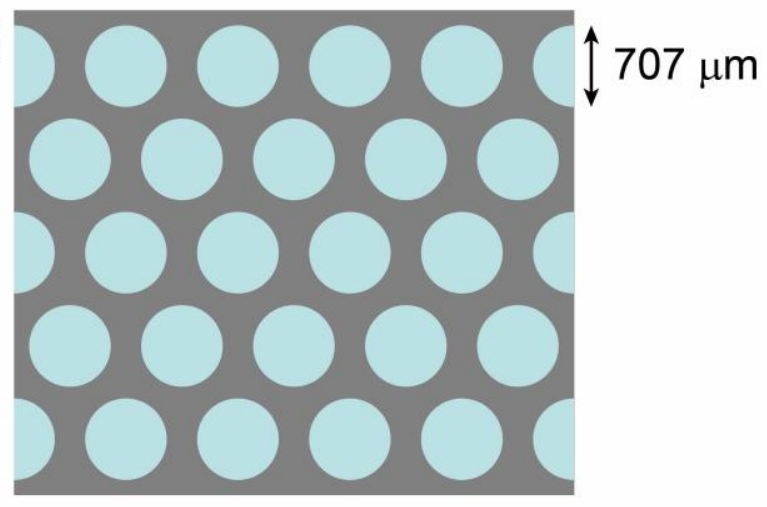

c)

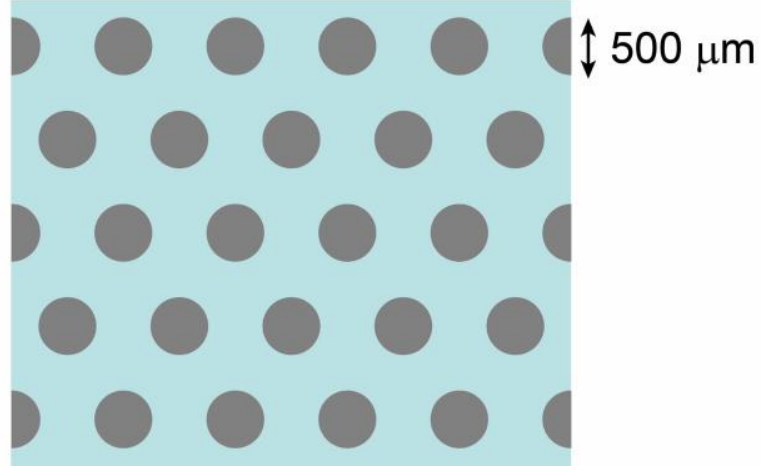

